
\title{
Research S Surate \\ Systematic THz Study of the Substrate Effect in Limiting the Mobility of Graphene
}

\section{Samantha Scarfe}

University of Ottawa

Wei Cui

University of Ottawa

Adina Luican-Mayer

University of Ottawa

Jean-Michel Ménard ( $\nabla$ jean-michel.menard@uottawa.ca )

University of Ottawa

\section{Research Article}

Keywords: Systematic THz Study, Graphene, spectroscopy

Posted Date: January 14th, 2021

DOI: https://doi.org/10.21203/rs.3.rs-144160/v1

License: (9) This work is licensed under a Creative Commons Attribution 4.0 International License.

Read Full License

Version of Record: A version of this preprint was published at Scientific Reports on April 22nd, 2021. See the published version at https://doi.org/10.1038/s41598-021-87894-5. 


\title{
Systematic THz study of the substrate effect in limiting the mobility of graphene
}

\author{
Samantha Scarfe, Wei Cui, Adina Luican-Mayer*, Jean-Michel Menard* \\ Department of Physics, University of Ottawa, Ottawa, Canada \\ luican-mayer@uottawa.ca \\ jean-michel.menard@uottawa.ca
}

We explore the substrate-dependent charge carrier dynamics of large area graphene films using contact-free non-invasive terahertz spectroscopy. The graphene samples are deposited on seven distinct substrates relevant to semiconductor technologies and flexible/photodetection devices. Using a Drude model for Dirac fermions in graphene and a fitting method based on statistical signal analysis, we extract transport properties such as the charge carrier density and carrier mobility. We find that graphene films supported by substrates with minimal charged impurities exhibit an enhanced carrier mobility, while substrates with a high surface roughness generally lead to a lower transport performance. The smallest amount of doping is observed for graphene placed on the polymer Zeonor, which also has the highest carrier mobility. This work provides valuable guidance in choosing an optimal substrate for graphene to enable applications where high mobility is required.

\section{Introduction}

Graphene, an atomic layer of carbon atoms arranged in a honeycomb lattice, is not only a rich playground for uncovering new physical phenomena, but also a potential component in future electronic and optoelectronic technologies ${ }^{1-3}$. For example, graphene has already been implemented into a broadband image sensor array for enhanced photodetection ${ }^{4}$ as well as memristors based on layered two-dimensional materials ${ }^{5}$. Graphene was also demonstrated to be a sensitive chemical and biological sensor ${ }^{6-8}$. With the development of large-scale production techniques such as epitaxial growth or chemical vapor deposition (CVD), large area graphene shows increasing promise for implementation into macroscale modern devices ${ }^{9}$.

Among its many properties, the high mobility of the massless Dirac fermions in graphene plays a key role in the implementation of high-speed electronic devices. Therefore, it is imperative to develop better understanding and control over the factors that affect scattering mechanisms in graphene films. Factors that limit large-area graphene's mobility can be either intrinsic or extrinsic $^{10}$. Intrinsic limiting factors include scattering from lattice defects, grain boundaries ${ }^{11-14}$, or phonons ${ }^{15,16}$. Due to the formation of grain boundaries and defects during the CVD growth, such scattering mechanisms could be more pronounced in large-area graphene compared to micron-scale mechanically exfoliated samples ${ }^{17,18}$. Among extrinsic limiting factors, scattering due to charged impurities is known to play a significant role ${ }^{19}$. Charged impurities can be present 
in the underlying substrate ${ }^{20}$, trapped between the film and substrate $e^{21}$, or on the surface of graphene in fabrication residues ${ }^{22}$.

Graphene supported by common substrates such as $\mathrm{SiO}_{2}, \mathrm{Si}_{3} \mathrm{~N}_{4}$, quartz or silicon is reported to have carrier mobilities varying between $500-10,000 \mathrm{~cm}^{2} \mathrm{~V}^{-1} \mathrm{~s}^{-1} 19,23-25$. Efforts to engineer substrates that minimize the presence of trapped charges and mechanical deformations led to three orders of magnitude improvement, with the highest mobilities (larger than $10^{6} \mathrm{~cm}^{2} /(\mathrm{Vs})$ ) reported in freely suspended devices ${ }^{26,27}$ or on top of atomically flat boron nitride $(\mathrm{BN})$ crystals $^{17,28}$. It is therefore critical that the graphene-substrate interaction be well understood for successful integration of large-area graphene films with high mobility. Substrates that ensure high mobility for graphene films could be exploited for improving device performance in future graphene integrated electronics.

To date, graphene-substrate interactions have been studied in devices by measuring the unpumped/non-excited carrier dynamics with both optical techniques and electrical transport techniques. For example, far-infrared spectroscopy (3-16 THz) was used to characterize graphene on different polar dielectric and organic polymer films. Graphene on hexamethyldisilazane (HMDS) notably exhibits a mobility four times higher than on $\mathrm{SiO}_{2}{ }^{29,30}$. Electrical transport was also used to investigate large-scale graphene deposited on several dielectrics commonly used in the semiconductor industry. The highest mobility was achieved when graphene was placed on $\mathrm{Si}_{3} \mathrm{~N}_{4}$, which outperformed $\mathrm{SiO}_{2}, \mathrm{HfO}_{2}, \mathrm{Al}_{2} \mathrm{O}_{3}$, and tetraethyl orthosilicate. This work indicated that carrier density fluctuation caused by the substrates is one of the main contributing factors for mobility degradation $^{31}$. Other studies relied on time-resolved $\mathrm{THz}$ spectroscopy to explore the substrate effect on graphene placed on sapphire, silicon, germanium, polyethylene naphthalate $(\mathrm{PEN})$, polyethylene terephthalate (PET), and quartz ${ }^{32,33}$. This technique provided information about the graphene DC conductivity, but the experimental spectral bandwidth, limited to frequencies up to $3 \mathrm{THz}$, could not resolve features attributed to carrier scattering. As a result, no information was obtained on carrier density or mobility. Finally, there has been more work that measured $^{23,34-36}$ and even spatially resolved ${ }^{24,25,37}$ the transport properties of wafer scale graphene using $\mathrm{THz}$ spectroscopy. These studies, however, are restricted to only one specific substrate, or do not investigate the substrate dependent dynamics at play.

Here, we perform time-domain terahertz transmission spectroscopy over a bandwidth extending from 0.5 to $5.5 \mathrm{THz}$ to measure graphene's transport properties when supported by substrates with different charged impurity densities, dielectric constants, and surface roughness. Our THz spectral window, extending beyond the limit of most commercial systems, is sufficiently broad to capture the spectral-dependent change in conductivity associated to the Drude roll-off frequency and therefore allows for measurement of both carrier density and Drude mobility. In comparison to other characterization methods based on electrical measurements, $\mathrm{THz}$ spectroscopy is a noninvasive technique that does not alter the graphene structure or rely on the deposition of electrical contacts, which composition and configuration often affect the measured transport parameters ${ }^{38}$. 
As such, this all-optical technique has a great potential to map out properties of large graphene sheets to optimize its integration into electronics or opto-electronics devices ${ }^{24,25,37}$.

The selection of substrates in this work is guided by the promise for graphene to be implemented into "beyond Moore" technologies ${ }^{1}$. We compare the mobility of graphene deposited on different materials: two gate dielectrics $\left(\mathrm{SiO}_{2}\right.$ and $\left.\mathrm{Si}_{3} \mathrm{~N}_{4}\right)$ which are traditional materials for computationalbased applications, silicon with three doping concentrations, and a hydrophobic, flexible, transparent polymer (Zeonor). We find that substrates with minimal amount of charged impurities and substrates that are flat generally exhibit the highest carrier mobilities. Notably, graphene on high-purity silicon and insulating Zeonor outperform the popular gate dielectrics. Our results guide the field of graphene integrated technologies, by providing valuable insights into the transport potential of graphene supported by substrates commonly used in devices.

\section{Results and Discussion}

In this work all samples are identically prepared, using graphene synthesized by CVD on $\mathrm{Cu}$ foil and transferred onto a substrate using a standard PMMA-based wet etching technique, schematically represented in Fig. 1a. A micrograph of transferred graphene on $\mathrm{SiO}_{2}$ in Fig. 1b shows continuous coverage across a large area. Atomic force microscopy (AFM) and Raman spectroscopy confirmed monolayer thickness as demonstrated by the height profile $(\sim 1 \mathrm{~nm})$ and Raman spectrum $\left(\mathrm{I}_{2 \mathrm{D}} / \mathrm{I}_{\mathrm{G}}=1.94\right)$ shown in Fig. 1c and Fig. 1d.

For the measurements, we employ a home-built time-domain terahertz spectrometer, schematically represented in Fig. 1e. The system is based on an optical source delivering 50 fs pulses centered at $1035 \mathrm{~nm}^{39}$. These pulses are focused onto a nonlinear GaP crystal where $\mathrm{THz}$ transients are generated by difference frequency mixing. The time-domain detection relies on electro-optic sampling inside a second $\mathrm{GaP}$ crystal $^{40}$, and the maximum signal sensitivity is found at $3.5 \mathrm{THz}$. The graphene sample, at room temperature and under normal ambient conditions, is placed at the $\mathrm{THz}$ focus and the measurement is performed in a transmission geometry. Note that we purge the system with dry air $(<0.5 \% \mathrm{RH})$ to minimize $\mathrm{THz}$ absorption in water vapor. Fourier transform of the time-domain data yields the complex field amplitude spectrum $\widetilde{E}_{S}$ transmitted through the graphene sample. As shown in Fig. 2 a, a reference field amplitude spectrum $\tilde{E}_{\text {ref }}$ is collected though the bare substrate, on a region without graphene, to calculate the complex transmission $\widetilde{T}=\widetilde{E}_{s} / \widetilde{E}_{\text {ref. }}$ Each measurement is repeated 10 times (Fig. 2c) under the same experimental conditions to obtain (i) an averaged signal with reduced noise and (ii) a frequency-dependent standard deviation indicative of the scan-to-scan experimental error.

The complex sheet conductance $\tilde{\sigma}_{s}$ can be straightforwardly extracted from $\tilde{T}$ considering graphene as an infinitely thin conducting film ${ }^{24}$, such that:

$$
\tilde{\sigma}_{s}=\frac{1+\tilde{n}_{s}}{Z_{0}}\left(\frac{1}{\tilde{T}}-1\right)
$$


where $\tilde{n}_{s}$ is the substrate refractive index and $Z_{0}$ the vacuum impedance (376.6 $\Omega$ ). Fig. $2 \mathrm{~b}$ shows a typical measurement and the corresponding transmission amplitude in the inset with error bars representing scan-to-scan uncertainties. Although Eq. 1 contains complex values, we do not consider the imaginary part of the conductivity in our analysis since this value can induce systematic errors as it is extremely sensitive to local variations of the substrate thickness. Therefore, we consider only the real part of the conductivity $\sigma_{S}$, which has proven to be sufficient to extract reliable graphene properties ${ }^{25}$. The frequency-dependent Drude model, which has been broadly used to describe carrier intra-band scattering behavior in graphene ${ }^{24,25,34-36}$, yields:

$$
\sigma_{S}=\frac{\sigma_{D C}}{1+\omega^{2} \tau^{2}}
$$

where $\sigma_{\mathrm{DC}}$ is the DC conductivity, $\omega$ is the radial frequency, and $\tau$ is the scattering time. As a result, we are able to extract values for both $\sigma_{\mathrm{DC}}$ and $\tau$ from the THz data. In general, the analysis of timedomain $\mathrm{THz}$ measurements is restricted to data points within a carefully selected spectral window centered around the maximum $\mathrm{THz}$ signal, where experimental noise is considered negligible. Here, we use instead a scan-to-scan statistics to include a larger bandwidth while error bars objectively determine the precision of each spectral data point. More specifically, experimental data are fitted to the Drude model (Eq. 2) with a weighting factor inversely proportional to the square of the frequency-dependent uncertainty. This quantitative approach ultimately allows more data points to be considered in the analysis and can therefore improve accuracy and reliability. To determine another type of uncertainty related to sample anisotropy, we collect data on more than 5 different positions on each sample and perform again the analysis described above (Fig. 2c). We rely on these multiple measurements and their standard deviation to evaluate a relative uncertainty of $\sigma_{\mathrm{DC}}$ and $\tau$, which is, in turn, used to calculate the relative uncertainty on other carrier transport parameters displayed in Figs. 3, 4 and 5. Finally, the full data acquisition process on each graphene sample is repeated at least twice on different days to test the reproducibility of our $\mathrm{THz}$ spectroscopy technique. This last step provides at least two data points, with their respective error bar, for all measured transport parameters and each substrate considered in our study.

We use the experimental procedure described above to obtain the parameters $\sigma_{\mathrm{DC}}$ and $\tau$ of graphene samples deposited on seven different substrates: three gate dielectrics on $\mathrm{Si}$ wafer $\left(\mathrm{Si}_{3} \mathrm{~N}_{4}\right.$, wet thermal grown $\mathrm{SiO}_{2}$, and dry thermal grown $\mathrm{SiO}_{2}$ ), $\mathrm{Si}$ with three doping concentrations corresponding to a resistivity of $10 \mathrm{k} \Omega$-cm (intrinsic doping), $15 \Omega$-cm (p-doped), and $5 \Omega$-cm (pdoped), and finally the flexible polymer Zeonor, a cyclo-olefin copolymer. We note that all bare substrates display high transparency to our broadband $\mathrm{THz}$ probe. Assuming a diffusive Drudetype transport regime ${ }^{41-44}$, we can then calculate graphene's carrier concentration $\left(N_{S}\right)$ and the mobility $(\mu)$ from the extracted $\sigma_{\mathrm{DC}}$ and $\tau$ using the following relations:

$$
\begin{gathered}
N_{S}=\frac{\pi \hbar^{2}}{v_{F}^{2} e^{4}}\left(\frac{\sigma_{D C}}{\tau}\right)^{2}, \\
\mu=\frac{\sigma_{D C}}{e N_{S}}=\frac{v_{F}^{2} e^{3}}{\pi \hbar^{2}} \frac{\tau^{2}}{\sigma_{D C}}=\frac{v_{F} e}{\sqrt{\pi} \hbar} \frac{\tau}{\sqrt{N_{S}}},
\end{gathered}
$$


where the Fermi velocity for graphene $v_{F} \approx 10^{6} \mathrm{~m} / \mathrm{s}$. As a result, $\mathrm{THz}$ measurements enable an effective and non-invasive method for probing the influence of the substrate on the transport properties of graphene films.

In Fig. 3 we plot the calculated parameters $N_{S}, \mu$, and $\tau$ for the seven different substrates described above. Fig. 3a shows that the carrier concentration $N_{S}$ in graphene is strongly dependent on the substrate. This variation, by more than an order of magnitude, can be attributed to the densities of free and trapped charges in the substrate, which have previously been identified as two dominant parameters affecting $N_{\mathrm{S}}{ }^{19,42}$. For the insulating substrates, trapped charges are particularly relevant, as those present at the surface may directly transfer charges to graphene and those beneath the surface may still capacitively induce charges in graphene. For the conductive substrates, $N_{\mathrm{S}}$ is largely due to direct charge transfer. In our experiment, the largest values of $N_{S}$ are obtained for graphene placed on doped $\mathrm{Si}$ and approach $10^{13} \mathrm{~cm}^{-2}$, corresponding to graphene's Fermi level being $350 \mathrm{meV}$ away from the Dirac point. This value is comparable to epitaxial graphene grown on $\mathrm{SiC}^{44}$. Insulating substrates, including typical gate dielectrics $\left(\mathrm{SiO}_{2}\right.$ and $\left.\mathrm{Si}_{3} \mathrm{~N}_{4}\right)$, high-resistivity Si $\left(\rho=10^{4} \Omega-\mathrm{cm}\right)$ and the polymer Zeonor $\left(\rho=10^{16} \Omega-\mathrm{cm}\right)$, lead to a lower $N_{\mathrm{S}}$. The lowest doping level we measured corresponds to graphene on Zeonor $\left(N_{\mathrm{S}}=8 \times 10^{11} \mathrm{~cm}^{-2}\right)$, indicating that this substrate has a minimal amount of free and trapped charges contributing to the doping of the 2D material on its surface.

Fig. $3 \mathrm{~b}$ shows the calculated mobilities for graphene films placed on the different substrates, presented in increasing order from left to right based on the calculated $N_{\mathrm{S}}$. We obtain higher mobilities for less doped samples, consistent with the notion that substrate charged impurities create an electrostatic potential that limits the mobility ${ }^{19,20}$. In particular, we find that graphene samples supported by high-resistivity $\mathrm{Si}$ and the insulating polymer Zeonor, show the highest mobilities $\mu \sim 5,000 \mathrm{~cm}^{2} \mathrm{~V}^{-1} \mathrm{~s}^{-1}$, more than an order of magnitude higher than graphene on Si $5 \Omega$ $\mathrm{cm}$ and $\mathrm{Si} 15 \Omega$-cm substrates. This indicates that boron impurities doping the Si crystals can act as scattering centers, reducing carrier mobility in the graphene film. Interestingly, graphene on high-resistivity Si shows a higher mobility than the one on gate dielectric substrates although they all possess trapped charges and have a similar carrier concentration $N_{\mathrm{S}}$. To understand this result, we note that a flat electrostatic potential profile, even if it causes charge doping, doesn't necessarily cause scattering. In the case of substrates with large amounts of trapped charges, their distribution can be non-uniform leading to more significant scattering ${ }^{46}$. This concept is supported by the measured scattering times $\tau$ shown in Fig. 1c. Most samples feature a $\tau$ around $40 \mathrm{fs}$, except for graphene on high-resistivity $\mathrm{Si}$, which exhibits a longer scattering time of $95 \mathrm{fs}$.

The dielectric constant $\kappa$ of the substrate or environment can play an important role in the transport properties of graphene; larger values of $\kappa$ imply the ability of an environment to readily screen charged potentials created by trapped scattering centers, and therefore enhance the carrier mobility ${ }^{48,49}$. In Fig. 4 , we plot the DC conductivity $\sigma_{\mathrm{DC}}$, as well as $\tau_{\text {scat }}, N_{\mathrm{S}}$, and $\mu$, as a function of the substrate's dielectric constant $\kappa$. We observe that three of these parameters: $\sigma_{\mathrm{DC}}, \tau$ and $N_{\mathrm{S}}$, 
generally increase with increasing $\kappa$, while the graphene's mobility $\mu$ does not appear to depend strongly on the substrate' dielectric constant. For the calculated $\tau$ and $\mu$ in Fig. $4 \mathrm{~b}$ and Fig. $4 \mathrm{~d}$, the value obtained for graphene placed on the doped Si substrate slightly departs from the general trend. We attribute this discrepancy to a strong inhomogeneous electrostatic potential created by the boron dopants in these substrates. Surprisingly, we observe that graphene on Zeonor exhibits the highest mobility, even though this substrate has the lowest dielectric constant among the samples measured. This indicates that the screening of charged impurities in the substrate is not necessarily the main contributor to the high mobility, but other factors come into play, such as the density of trapped and free charges in the substrate, as discussed previously.

Although much less systematically studied, substrate roughness can also affect the carrier mobility in graphene since a smooth interface contributes to reduced strain of the film or eliminates wrinkles and edges that could induce scattering. Using atomic force microscopy, we measure the roughness $\left(R_{\mathrm{q}}\right)$ of the seven substrates used in this experiment. In general, we observe high carrier mobility for smooth substrates (small values of $\left.R_{\mathrm{q}}\right)$. For example, $\mathrm{Si}-10 \mathrm{k} \Omega-\mathrm{cm}\left(R_{\mathrm{q}}=220 \mathrm{pm}\right)$ out-performs wet $\mathrm{SiO}_{2}\left(R_{\mathrm{q}}=380 \mathrm{pm}\right)$. However, we note two exceptions in our experiment: (i) graphene on doped Si features a low mobility although the substrate is relatively smooth. This suggests that Coulomb scattering is a dominant mechanism in this case. (ii) At the other extreme, graphene on Zeonor has one of the highest mobilities in our study while the substrate roughness is higher, $R_{\mathrm{q}}=650 \mathrm{pm}$. To assess the adhesion of graphene to Zeonor, we measured the roughness of sample when graphene was placed on Zeonor, which shows a smoother morphology $\left(R_{\mathrm{q}}=520 \mathrm{pm}\right)$, suggesting relatively poor adhesion of the graphene to the substrate. As a result, graphene may locally be suspended and experience a reduced influence from the substrate. This characteristic combined with the indication of minimal trapped charges at the graphene-Zeonor interface (see Fig. 3a and related discussion), explains the measured high mobility. The outperformance of graphene on a hydrophobic polymer compared to a gate dielectric is consistent with previous reports where graphene field effect transistors on parylene ${ }^{50}$ and $\operatorname{HMDS}^{29,30}$ demonstrated reduced ambient doping and hysteresis compared to those on $\mathrm{SiO}_{2}$.

\section{Conclusion}

In summary, we have demonstrated the implementation of terahertz transmission spectroscopy for probing the equilibrium transport properties of CVD graphene supported by seven substrates, distinct in their density of charged impurities, dielectric constant and surface roughness. We find that the resulting conductance spectra for graphene films agrees well to a Drude model for intraband behavior, consistent with other reports. We have demonstrated a method for weighted Drude fitting based on statistical averaging and extracted transport properties $\left(N_{S}, \mu, \tau\right.$, and $\left.\sigma_{\mathrm{DC}}\right)$, for graphene supported by distinct substrates. When comparing the results on different substrates, we find the highest mobility for graphene placed on pure silicon and the polymer Zeonor, which outperformed commonly used gate dielectrics. This highlights that the densities of trapped and free charged impurities in the substrate are likely key limiting factors of the graphene film's mobility. We also find the mobility of devices to be generally improved for substrates that are 
smoother, except for graphene on Zeonor. In this case, we interpret the high mobility to be due to both the poor adhesion between Zeonor and graphene, minimizing the substrate effect, and to the low density of trapped charged impurities at the interface. A flexible polymer, therefore, can be considered as a promising alternative to some gate dielectrics. These results provide valuable guidance for the effective integration of graphene into technological platforms where high mobility is necessary.

\section{ACKNOWLEDGEMENTS}

We would like to acknowledge Prof. Xu Du and Prof. Pawel Hawrylak for insightful discussion and Dr. Lauren Gingras for technical help and support. SS, AL-M, and J-MM acknowledge funding through DND-IDEaS (IDEaS-1-1A-CP-0122). AL-M acknowledges funding from the National Sciences and Engineering Research Council (NSERC) Discovery Grant RGPIN-201606717. J-MM acknowledges funding from the National Sciences and Engineering Research Council (NSERC) Discovery Grant RGPIN-2016-04797 and the Canada Foundation for Innovation (CFI) (Project number 35269).

\section{AUTHOR CONTRIBUTIONS}

SS fabricated the samples, took the data, analyzed the data, and wrote the manuscript. WC took data and participated in the manuscript presentation. AL-M and J-MM coordinated the project and wrote the manuscript. All authors discussed the data and its interpretation.

\section{RFERENCES}

1 Akinwande, D. et al. Graphene and two-dimensional materials for silicon technology. Nature 573, 507-518, doi:10.1038/s41586-019-1573-9 (2019).

2 lannaccone, G., Bonaccorso, F., Colombo, L. \& Fiori, G. Quantum engineering of transistors based on 2D materials heterostructures. Nature Nanotechnology 13, 183-191, doi:10.1038/s41565-018-0082-6 (2018).

3 Romagnoli, M. et al. Graphene-based integrated photonics for next-generation datacom and telecom. Nature Reviews Materials 3, 392-414, doi:10.1038/s41578-018-0040-9 (2018).

4 Goossens, S. et al. Broadband image sensor array based on graphene-CMOS integration. Nature Photonics 11, 366-371, doi:10.1038/nphoton.2017.75 (2017).

5 Wang, M. et al. Robust memristors based on layered two-dimensional materials. Nature Electronics 1, 130-136, doi:10.1038/s41928-018-0021-4 (2018).

6 Rautela, R. et al. Mechanistic Insight into the Limiting Factors of Graphene-Based Environmental Sensors. ACS Applied Materials \& Interfaces, doi:10.1021/acsami.0c09051 (2020).

7 Schedin, F. et al. Detection of individual gas molecules adsorbed on graphene. Nature Materials 6, 652-655, doi:10.1038/nmat1967 (2007).

$8 \quad$ Fakih, l. et al. Selective ion sensing with high resolution large area graphene field effect transistor arrays. Nature Communications 11, 3226, doi:10.1038/s41467-020-16979-y (2020).

9 Zurutuza, A. \& Marinelli, C. Challenges and opportunities in graphene commercialization. Nature Nanotechnology 9, 730-734, doi:10.1038/nnano.2014.225 (2014). 
Bolotin, K. I. Graphene (eds Viera Skákalová \& Alan B. Kaiser) 199-227 (Woodhead Publishing, 2014).

11 Bøggild, P. et al. Mapping the electrical properties of large-area graphene. 2D Materials 4, 042003, doi:10.1088/2053-1583/aa8683 (2017).

12 Huang, P. Y. et al. Grains and grain boundaries in single-layer graphene atomic patchwork quilts. Nature 469, 389-392, doi:10.1038/nature09718 (2011).

13 Luican-Mayer, A. et al. Localized electronic states at grain boundaries on the surface of graphene and graphite. 2D Materials 3, 031005, doi:10.1088/2053-1583/3/3/031005 (2016). Luican-Mayer, A., Li, G. \& Andrei, E. Y. Atomic scale characterization of mismatched graphene layers. Journal of Electron Spectroscopy and Related Phenomena 219, 92-98, doi:https://doi.org/10.1016/i.elspec.2017.01.005 (2017). Castro, E. V. et al. Limits on Charge Carrier Mobility in Suspended Graphene due to Flexural Phonons. Physical Review Letters 105, 266601, doi:10.1103/PhysRevLett.105.266601 (2010). Morozov, S. V. et al. Giant Intrinsic Carrier Mobilities in Graphene and Its Bilayer. Physical Review Letters 100, 016602, doi:10.1103/PhysRevLett.100.016602 (2008).

17 Petrone, N. et al. Chemical Vapor Deposition-Derived Graphene with Electrical Performance of Exfoliated Graphene. Nano Letters 12, 2751-2756, doi:10.1021/nl204481s (2012).

18 Emtsev, K. V. et al. Towards wafer-size graphene layers by atmospheric pressure graphitization of silicon carbide. Nat Mater 8, 203-207, doi:10.1038/nmat2382 (2009).

19 Chen, J. H. et al. Charged-impurity scattering in graphene. Nature Physics 4, 377-381, doi:10.1038/nphys935 (2008).

20 Burson, K. M. et al. Direct Imaging of Charged Impurity Density in Common Graphene Substrates. Nano Letters 13, 3576-3580, doi:10.1021/nl4012529 (2013).

21 Boddison-Chouinard, J., Scarfe, S., Watanabe, K., Taniguchi, T. \& Luican-Mayer, A. Flattening van der Waals heterostructure interfaces by local thermal treatment. Applied Physics Letters 115, 231603, doi:10.1063/1.5131022 (2019).

22 Ishigami, M., Chen, J. H., Cullen, W. G., Fuhrer, M. S. \& Williams, E. D. Atomic Structure of Graphene on SiO2. Nano Letters 7, 1643-1648, doi:10.1021/nl070613a (2007).

23 Yan, H. et al. Infrared Spectroscopy of Wafer-Scale Graphene. ACS Nano 5, 9854-9860, doi:10.1021/nn203506n (2011).

24 Buron, J. D. et al. Graphene mobility mapping. Scientific Reports 5, 12305, doi:10.1038/srep12305 (2015).

25 Buron, J. D. et al. Terahertz wafer-scale mobility mapping of graphene on insulating substrates without a gate. Opt. Express 23, 30721-30729, doi:10.1364/OE.23.030721 (2015).

26 Du, X., Skachko, I., Barker, A. \& Andrei, E. Y. Approaching ballistic transport in suspended graphene. Nature Nanotechnology 3, 491-495, doi:10.1038/nnano.2008.199 (2008). Bolotin, K. I. et al. Ultrahigh electron mobility in suspended graphene. Solid State Communications 146, 351-355, doi:https://doi.org/10.1016/j.ssc.2008.02.024 (2008).

28 Dean, C. R. et al. Boron nitride substrates for high-quality graphene electronics. Nature Nanotechnology 5, 722-726, doi:10.1038/nnano.2010.172 (2010). Lafkioti, M. et al. Graphene on a Hydrophobic Substrate: Doping Reduction and Hysteresis Suppression under Ambient Conditions. Nano Letters 10, 1149-1153, doi:10.1021/nl903162a (2010).

30 Kim, J. Y. et al. Far-infrared study of substrate-effect on large scale graphene. Applied Physics Letters 98, 201907, doi:10.1063/1.3590773 (2011).

$31 \mathrm{Hu}$, Z., Sinha, D. P., Lee, J. U. \& Liehr, M. Substrate dielectric effects on graphene field effect transistors. Journal of Applied Physics 115, 194507, doi:10.1063/1.4879236 (2014). 
Lin, H. et al. Contactless graphene conductivity mapping on a wide range of substrates with terahertz time-domain reflection spectroscopy. Scientific Reports 7, 10625, doi:10.1038/s41598017-09809-7 (2017).

33 Gabriel, D., Sempere, B., Colominas, C. \& Ferrer-Anglada, N. THz-conductivity of CVD graphene on different substrates. physica status solidi (b) 252, 2423-2428, doi:https://doi.org/10.1002/pssb.201552248 (2015).

34 Buron, J. D. et al. Electrically Continuous Graphene from Single Crystal Copper Verified by Terahertz Conductance Spectroscopy and Micro Four-Point Probe. Nano Letters 14, 6348-6355, doi:10.1021/nl5028167 (2014).

35 Ren, L. et al. Terahertz and Infrared Spectroscopy of Gated Large-Area Graphene. Nano Letters 12, 3711-3715, doi:10.1021/nl301496r (2012).

36 Tomaino, J. L. et al. Terahertz imaging and spectroscopy of large-area single-layer graphene. Opt. Express 19, 141-146, doi:10.1364/OE.19.000141 (2011).

37 Whelan, P. R. et al. Conductivity mapping of graphene on polymeric films by terahertz timedomain spectroscopy. Opt. Express 26, 17748-17754, doi:10.1364/OE.26.017748 (2018). Liu, W., Wei, J., Sun, X. \& Yu, H. A Study on graphene - metal contact. Crystals 3, 257, doi:10.3390/cryst3010257 (2013) Cui, W. et al. Broadband and tunable time-resolved THz system using argon-filled hollow-core photonic crystal fiber. APL Photonics 3, 111301, doi:10.1063/1.5043270 (2018). Halpin, A. et al. Enhanced Terahertz Detection Efficiency via Grating-Assisted Noncollinear Electro-Optic Sampling. Physical Review Applied 12, 031003, doi:10.1103/PhysRevApplied.12.031003 (2019).

41 Horng, J. et al. Drude conductivity of Dirac fermions in graphene. Physical Review B 83, 165113, doi:10.1103/PhysRevB.83.165113 (2011).

42 Hwang, E. H., Adam, S. \& Sarma, S. D. Carrier Transport in Two-Dimensional Graphene Layers. Physical Review Letters 98, 186806, doi:10.1103/PhysRevLett.98.186806 (2007). Das Sarma, S., Adam, S., Hwang, E. H. \& Rossi, E. Electronic transport in two-dimensional graphene. Reviews of Modern Physics 83, 407-470, doi:10.1103/RevModPhys.83.407 (2011). Ando, T. Screening Effect and Impurity Scattering in Monolayer Graphene. Journal of the Physical Society of Japan 75, 074716, doi:10.1143/JPSJ.75.074716 (2006).

45 Riedl, C., Coletti, C., Iwasaki, T., Zakharov, A. A. \& Starke, U. Quasi-Free-Standing Epitaxial Graphene on SiC Obtained by Hydrogen Intercalation. Physical Review Letters 103, 246804, doi:10.1103/PhysRevLett.103.246804 (2009).

46 Martin, J. et al. Observation of electron-hole puddles in graphene using a scanning singleelectron transistor. Nature Physics 4, 144-148, doi:10.1038/nphys781 (2008).

47 Jena, D. \& Konar, A. Enhancement of Carrier Mobility in Semiconductor Nanostructures by Dielectric Engineering. Physical Review Letters 98, 136805, doi:10.1103/PhysRevLett.98.136805 (2007).

48 Jang, C. et al. Tuning the Effective Fine Structure Constant in Graphene: Opposing Effects of Dielectric Screening on Short- and Long-Range Potential Scattering. Physical Review Letters 101, 146805, doi:10.1103/PhysRevLett.101.146805 (2008).

49 Newaz, A. K. M., Puzyrev, Y. S., Wang, B., Pantelides, S. T. \& Bolotin, K. I. Probing charge scattering mechanisms in suspended graphene by varying its dielectric environment. Nature Communications 3, 734, doi:10.1038/ncomms1740 (2012).

50 Sabri, S. S. et al. Graphene field effect transistors with parylene gate dielectric. Applied Physics Letters 95, 242104, doi:10.1063/1.3273396 (2009). 
(a)

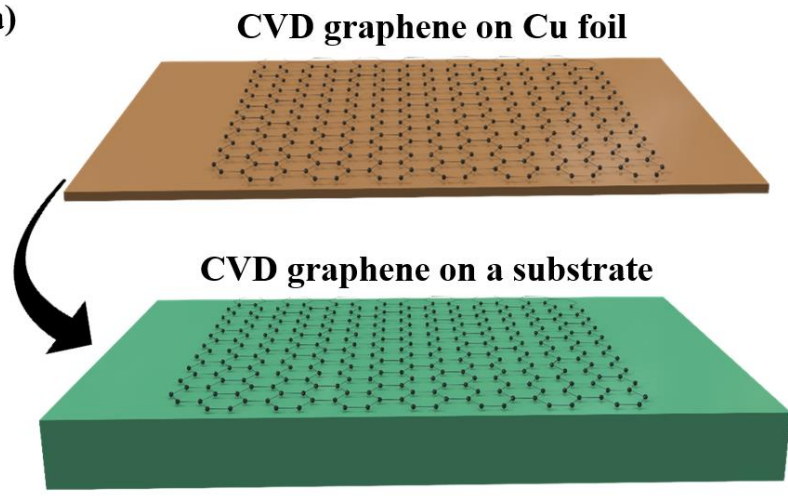

(b)

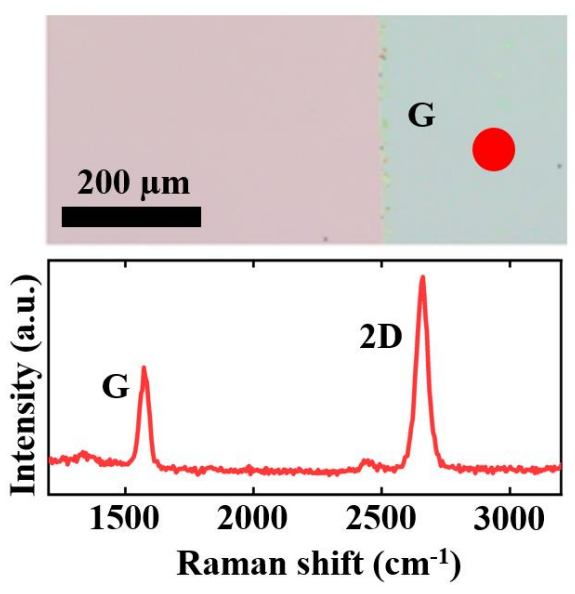

(d)
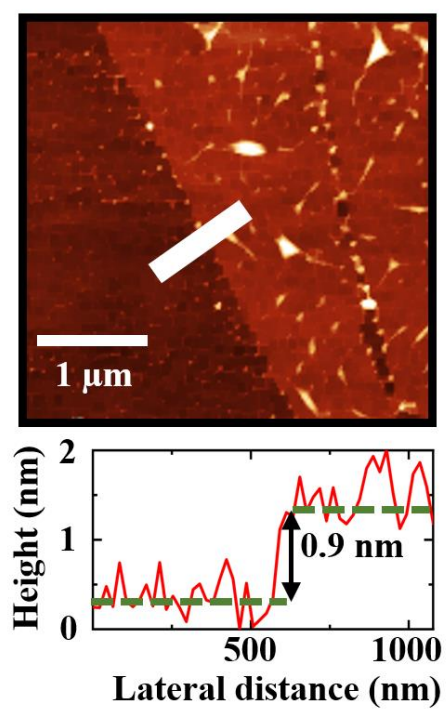

(e)
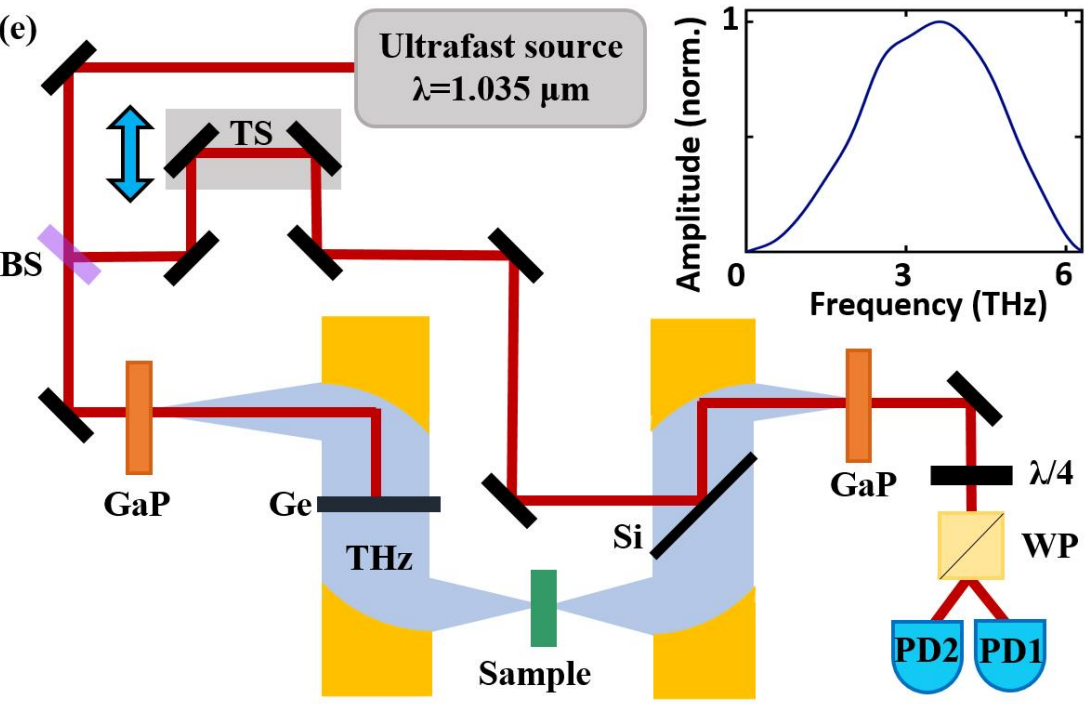

BS

(c)

Figure 1: Sample fabrication and experimental set-up. (a) Schematic of sample fabrication: $\mathrm{CVD}$ graphene on $\mathrm{Cu}$ foil is transferred onto a desired substrate. (b) 5X optical microscope image of graphene (right section) on $\mathrm{SiO}_{2}$ (left section). (c) Raman spectrum of a transferred graphene film featuring distinctive $\mathrm{G}$ and $2 \mathrm{D}$ peaks. (d) Atomic force micrograph of graphene transferred on $\mathrm{SiO}_{2}$ together with a height profile across the graphene edge, indicated by the white line. (e) Schematic white line. (e) Schematic of the time-domain terahertz spectrometer with its optical components: beam splitter (BS), translation stage (TS), gallium phosphide crystals $(\mathrm{GaP})$, germanium and silicon wafer ( $\mathrm{Ge}$ and $\mathrm{Si}$ ), quarter wave plate (QWP), Wollaston prism (WP), and balanced photodetectors (PD). 

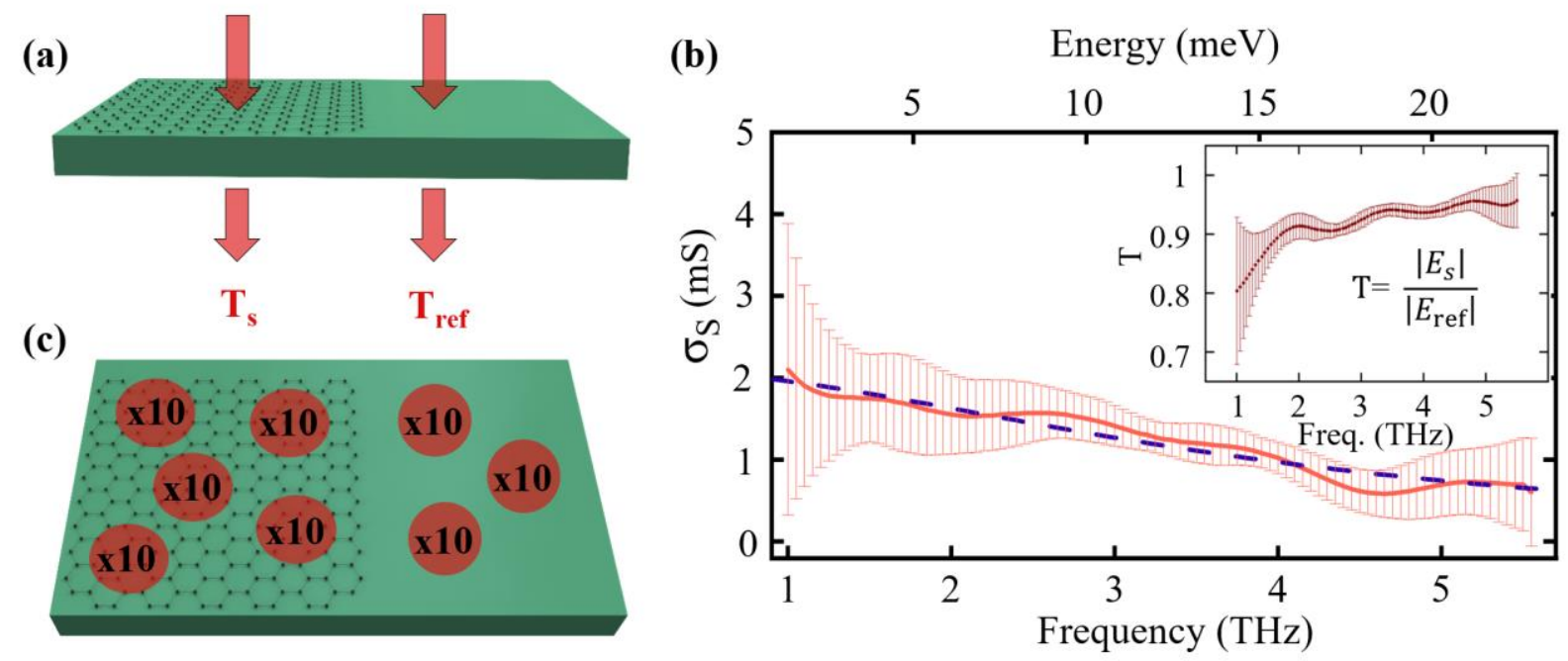

Figure 2: Description of the THz spectroscopy experimental procedure. (a) Geometry of the $\mathrm{THz}$ reference and sample measurement. (b) The real sheet conductance is extracted by comparing the transmission through the reference and sample (inset). Solid line represents experimental data and the error; dashed line represents the error weighted Drude fit to the data. (c) Description of the data collected for signal averaging and estimation of uncertainty: 10 scans are taken at a single spot on the graphene sample, indicated by a red dot. This is repeated across the sample at different spots at least 5 times, indicated by the several red dots. 

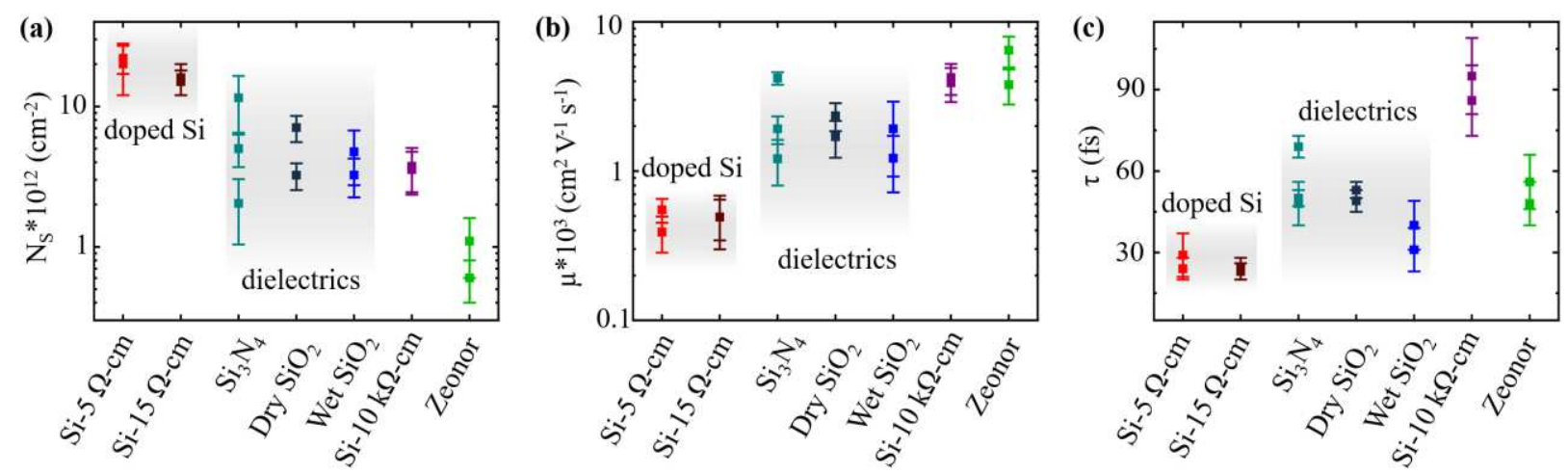

Figure 3: Results obtained for seven distinct substrates by applying the Drude fit to our experimental conductance spectra. The calculated transport parameters are: (a) carrier density $\mathrm{N}_{S}$, (b) Drude carrier mobility $\mu$, and (c) scattering time $\tau$. Solid squares represent the average value measured across several spots on a sample. Error bars represent one standard deviation. Measurements are repeated at least twice for each sample on a different day under similar experimental conditions. 

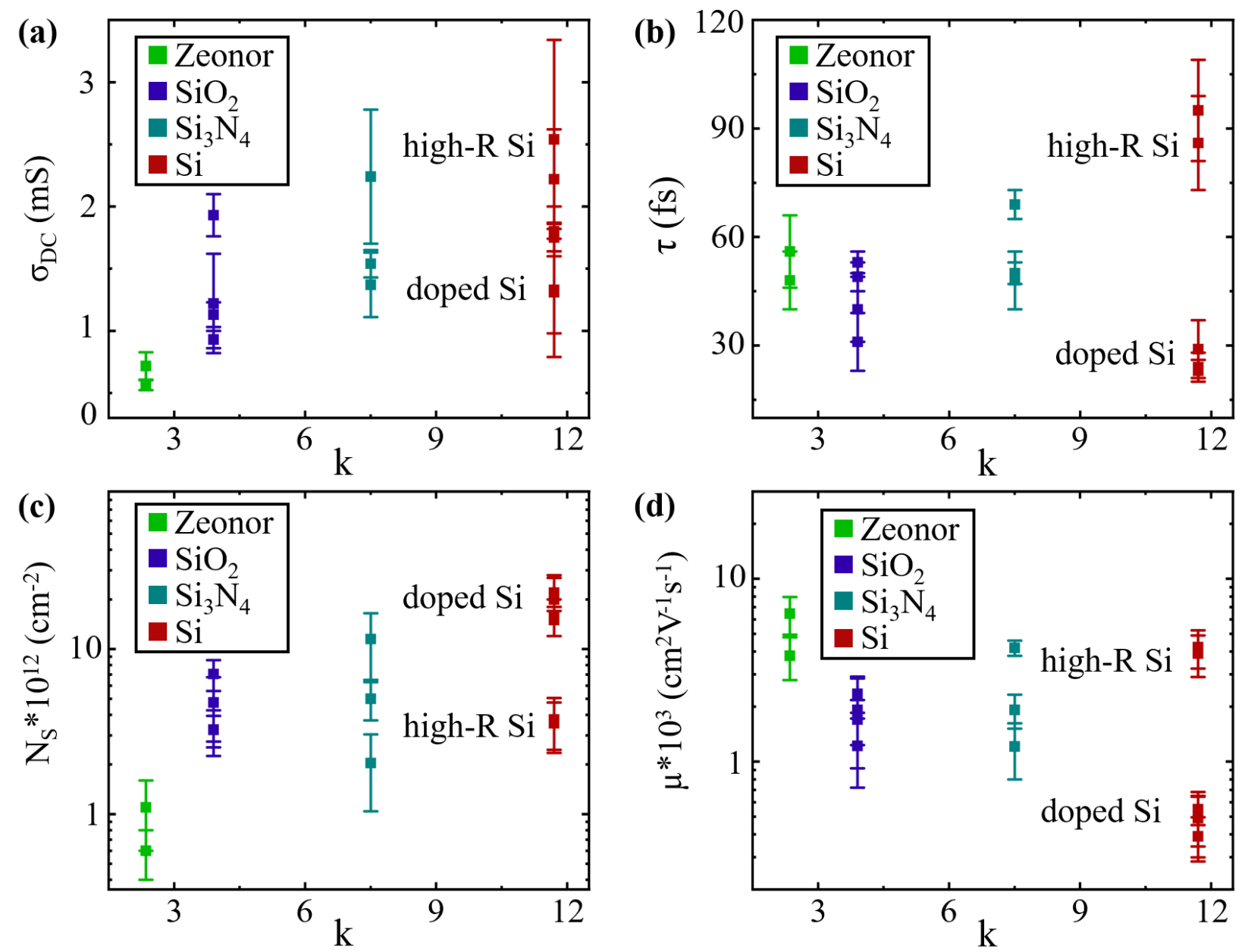

Figure 4: Experimentally measured transport parameters plotted as a function of the substrate dielectric constant $\kappa$ : (a) DC conductivity $\sigma_{D C}$, (b) scattering time $\tau$, (c) carrier density $N_{S}$, and (d) mobility $\mu$. 


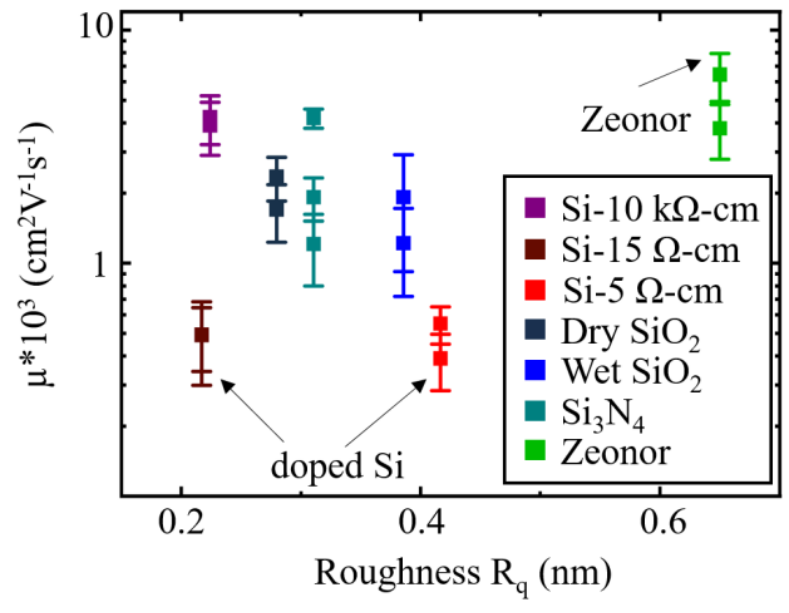

Figure 5: Mobility of graphene devices supported by substrates with different surface roughness. 
(a)

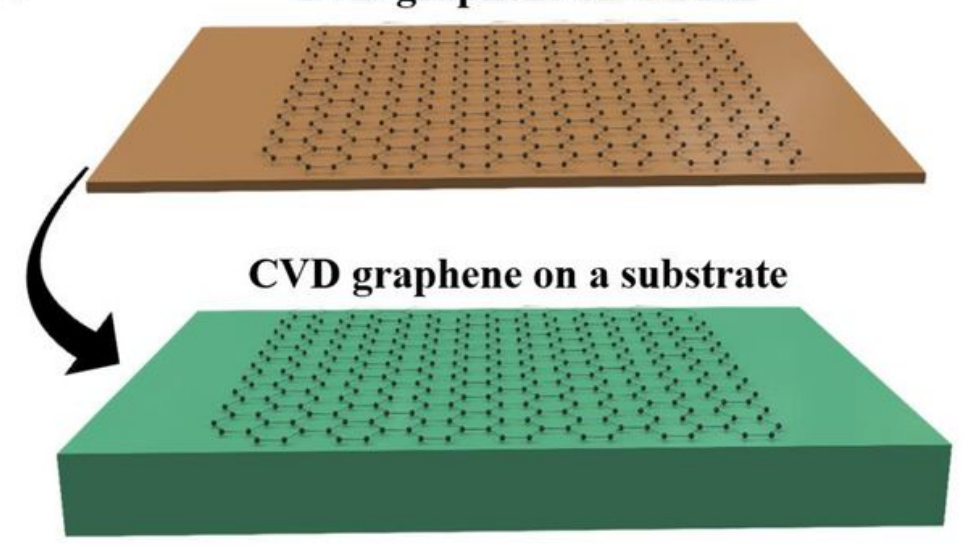

(b)

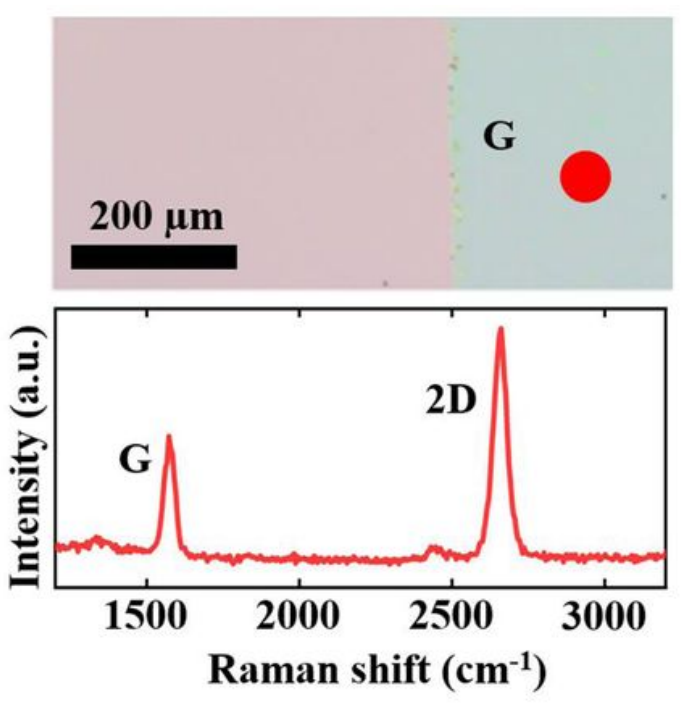

(c) (d)
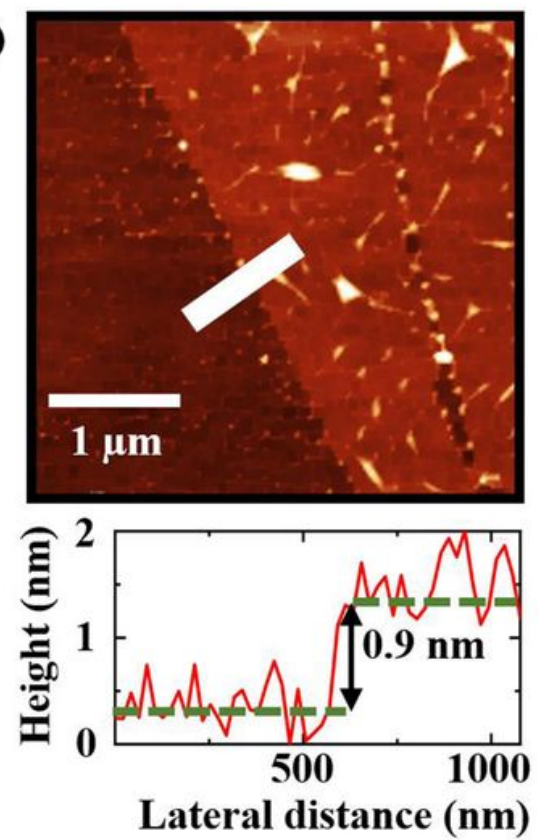

(e)

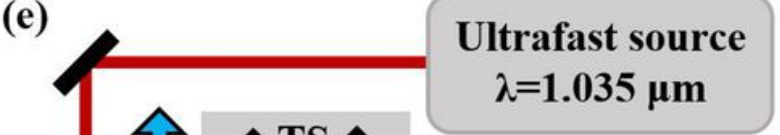

BS

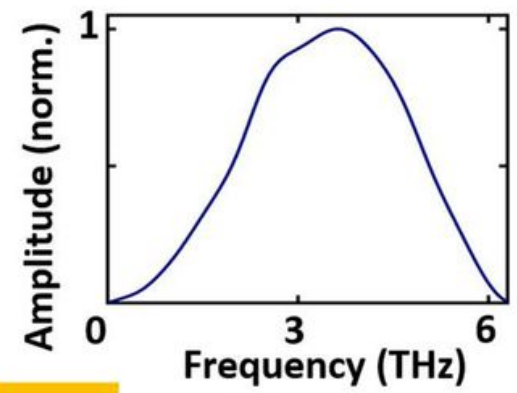

GaP

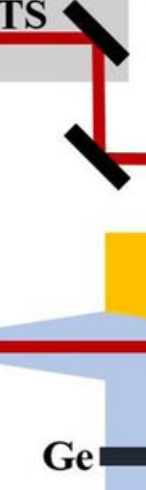

$\mathrm{THz}$

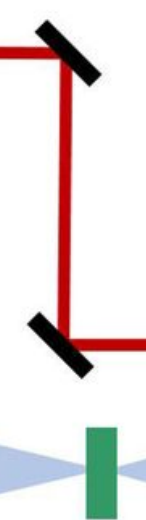

Sample

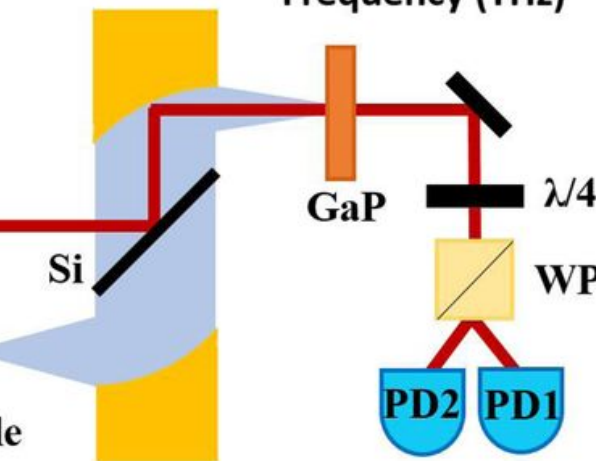

\section{Figure 1}

Sample fabrication and experimental set-up. (a) Schematic of sample fabrication: CVD graphene on Cu foil is transferred onto a desired substrate. (b) $5 \mathrm{X}$ optical microscope image of graphene (right section) on $\mathrm{SiO} 2$ (left section). (c) Raman spectrum of a transferred graphene film featuring distinctive $\mathrm{G}$ and 2D peaks. (d) Atomic force micrograph of graphene transferred on SiO2 together with a height profile across the graphene edge, indicated by the white line. (e) Schematic white line. (e) Schematic of the time-domain terahertz spectrometer with its optical components: beam splitter (BS), translation stage (TS), gallium phosphide crystals (GaP), germanium and silicon wafer (Ge and $\mathrm{Si}$ ), quarter wave plate (QWP), Wollaston prism (WP), and balanced photodetectors (PD). 

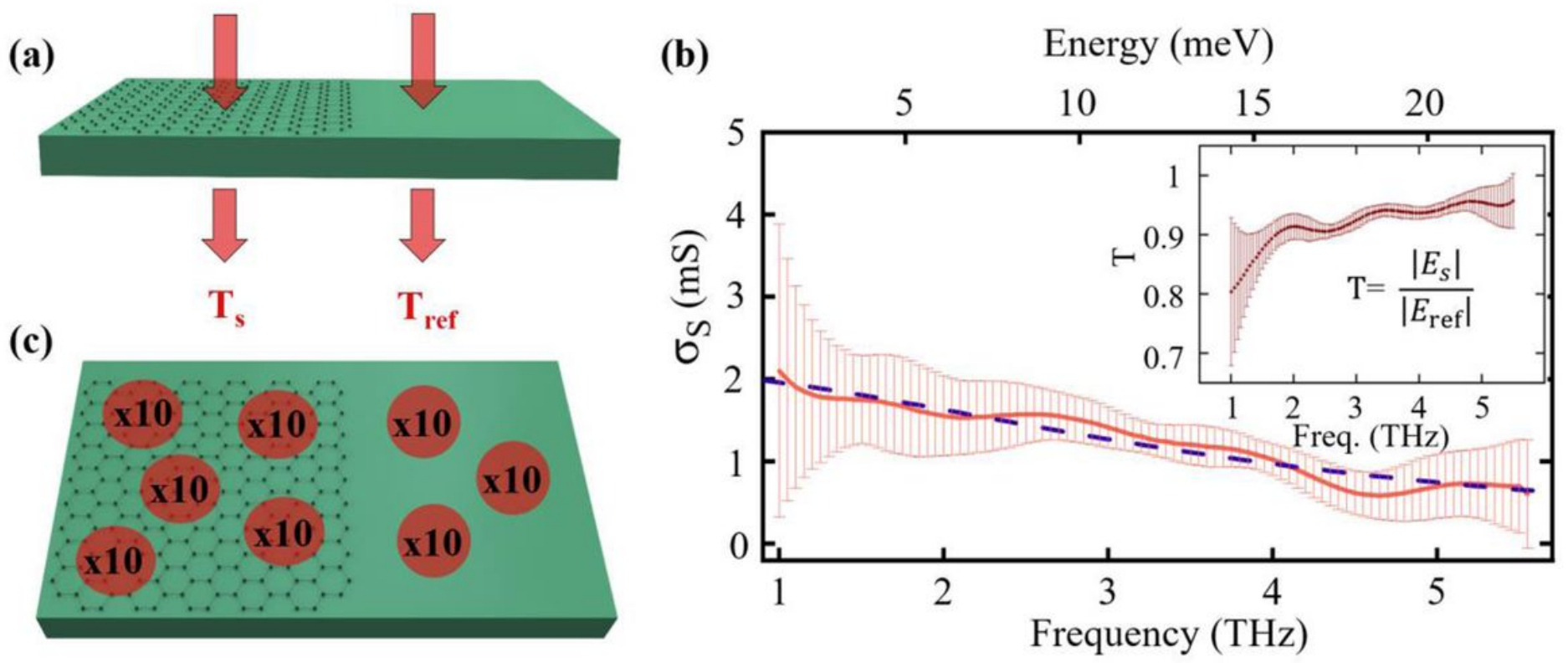

Figure 2

Description of the THz spectroscopy experimental procedure. (a) Geometry of the THz reference and sample measurement. (b) The real sheet conductance is extracted by comparing the transmission through the reference and sample (inset). Solid line represents experimental data and the error; dashed line represents the error weighted Drude fit to the data. (c) Description of the data collected for signal averaging and estimation of uncertainty: 10 scans are taken at a single spot on the graphene sample, indicated by a red dot. This is repeated across the sample at different spots at least 5 times, indicated by the several red dots.
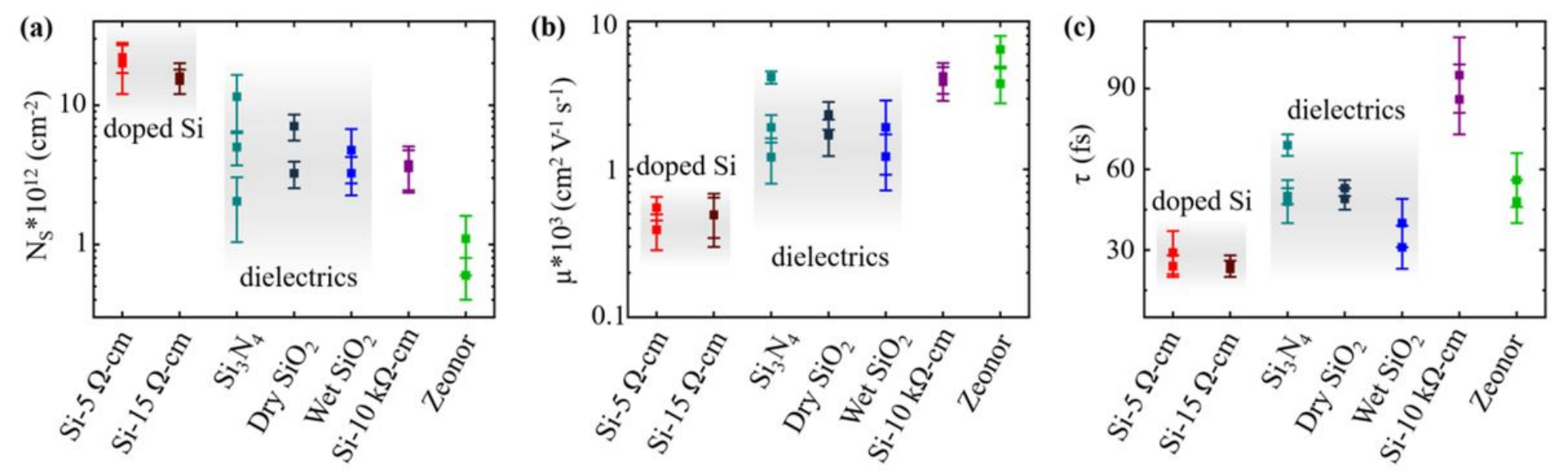

\section{Figure 3}

Results obtained for seven distinct substrates by applying the Drude fit to our experimental conductance spectra. The calculated transport parameters are: (a) carrier density NS, (b) Drude carrier mobility $\mu$, and (c) scattering time $\tau$. Solid squares represent the average value measured across several spots on a 
sample. Error bars represent one standard deviation. Measurements are repeated at least twice for each sample on a different day under similar experimental conditions.
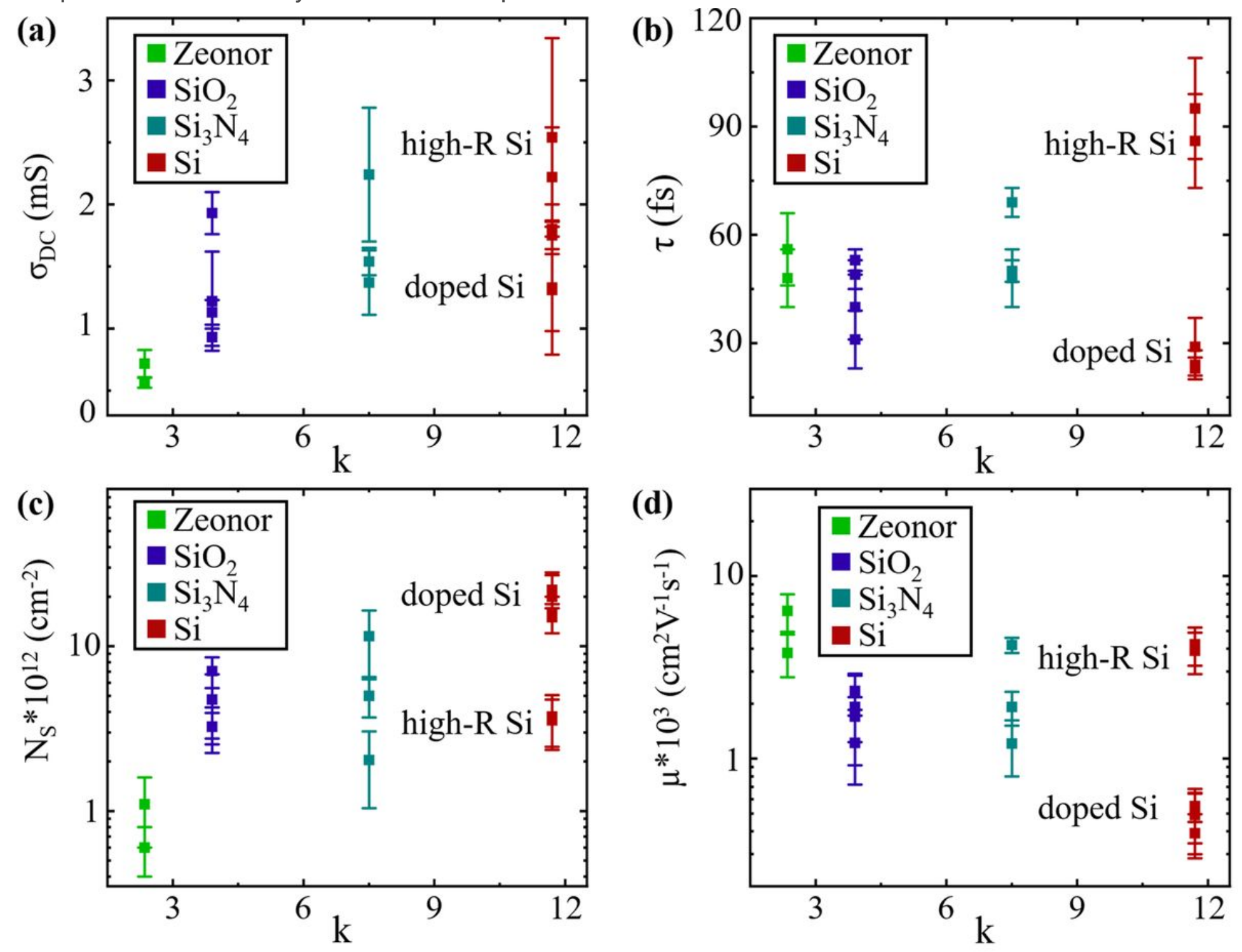

Figure 4

Experimentally measured transport parameters plotted as a function of the substrate dielectric constant $\kappa$ : (a) DC conductivity $\sigma D C$, (b) scattering time $\tau$, (c) carrier density NS, and (d) mobility $\mu$. 


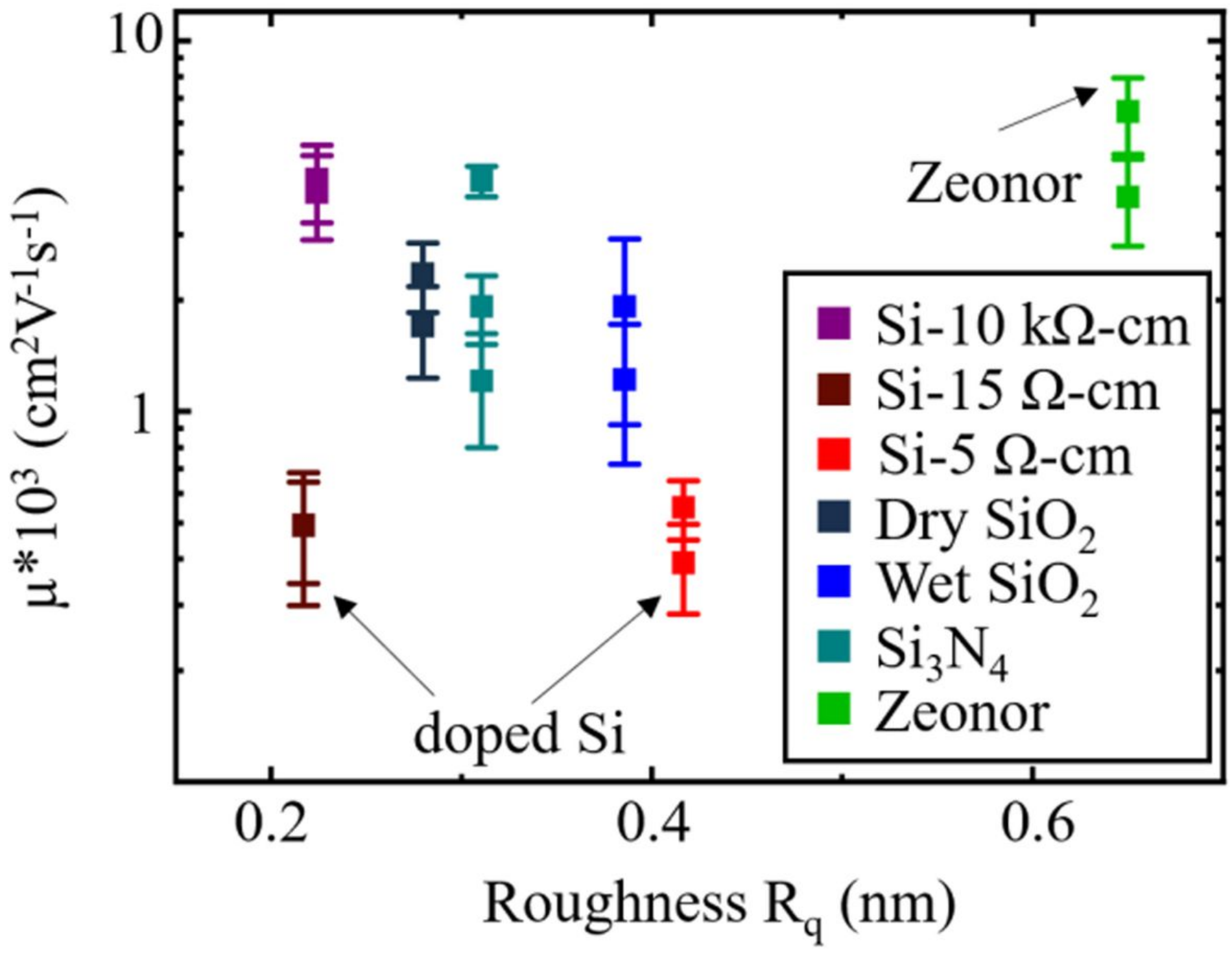

Figure 5

Mobility of graphene devices supported by substrates with different surface roughness. 